\title{
A Rigorous Upper Bound in Electrostatics on a Random Lattice Ensemble ${ }^{\star}$
}

\author{
R. Friedberg and S. Yancopoulos \\ Columbia University, New York, NY 10027, USA
}

\begin{abstract}
We consider a Kirchhoff network on a random two-dimensional lattice with links and weights as previously specified, and a circular boundary of radius $R$. We show rigorously that the resistance between the central point and the boundary, averaged over all placements of the remaining sites with site density $\varrho$, is bounded above by
\end{abstract}

$$
\begin{aligned}
& (4 \pi)^{-1}\left[\ln \left(4 \pi \varrho R^{2}\right)+1\right]+16\left[\tan ^{-1} 5^{-1 / 4}+5^{1 / 4} /(\sqrt{5}+1)^{2}\right] \\
& \quad \simeq(4 \pi)^{-1} \ln \left(4 \pi \varrho R^{2}\right)+12.0 .
\end{aligned}
$$

\section{Introduction}

In a series of recent papers [1-3], a formulation of field theory on a random lattice has been developed. The possibility was raised that this approach might lead to a true theory of nature and not merely a method of making numerical approximations to continuous field theory. According to this view, physical quantities might be obtained as averages over an ensemble of random lattices.

Such an idea invites a program of research in which almost every branch of mathematical physics may be examined to see how it is altered when reformulated along the lines of [1-3]. We have addressed ourselves here to electrostatics in two dimensions, and specifically to the finiteness of the self-potential of a charge.

When electrostatics in a vacuum is reformulated as a lattice theory, it becomes the problem of a network of capacitors joined at nodes which may bear charge. If a unit charge is placed at the origin, the potential at large distances will behave as in the continuous theory, but at short distances it will behave quite differently. In the continuous theory, the potential is singular at the origin; in the network, it remains finite even at the node on which the charge is placed. (In two dimensions, we make the potential vanish at a finite boundary so as to eliminate the logarithmic infinity at large distances.)

* This research was supported in part by the U.S. Department of Energy 
By a well-known analogy, the capacitors may be replaced by resistors, charge by current, and energy by power. The finite quantity in question is then the resistance $g$ between a single node and the boundary of the system. This quantity has no finite analog in the continuous theory.

Since $g$ is different for each lattice, we expect to find a universal constant $\langle g\rangle$ by averaging over all distributions of lattice sites with a given average density $\varrho$. In two dimensions $g$ is dimensionless, and therefore the result will be independent of $\varrho$. But here a question presents itself. The ensemble includes some lattices whose density near the origin is very much greater than $\varrho$. For these lattices, $g$ will be correspondingly large, since the network acts like a continuum roughly down to the lattice spacing. Thus $g$ can be arbitrarily large, for improbable lattices. One may ask whether $\langle g\rangle$ is nevertheless finite.

Taking the rules for linking lattice sites from [1], and for assigning a conductance to each link from [3], we have answered this question affirmatively in two dimensions, by deriving a rigorous upper bound for $\langle g\rangle$. In Sect. 2 we formulate the problem. In Sect. 3 we show that for any lattice, $g$ does not exceed the sum of two quantities $g_{1}$ and $g_{2}$, defined simply from the immediate neighborhood of the site at the origin. In Sects. 4 and 5 we derive upper bounds for $\left\langle g_{1}\right\rangle$ and $\left\langle g_{2}\right\rangle$. In Sect. 6 we assemble and discuss the results.

\section{Formulation of Problem}

We consider a circle of radius $R$, at the center of which we place a permanent lattice site, $p_{0}$. We distribute the remaining sites independently throughout the area $\pi R^{2}$, with average density $\varrho$. That is, the probability of finding a site in an infinitesimal area $d A$ is $\varrho d A$, regardless of the position of other sites. This yields an ensemble of position distributions identical (apart from the site $p_{0}$ ) to that derived from the grand canonical ensemble for a classical ideal gas.

We link sites according to the prescription of [1]: a polygonal cell is defined for each site so that each point on the boundary between two cells is equidistant from the two sites in question. Two sites are linked if their cells share a boundary. The links between sites then form a network of triangles. (An equivalent prescription, ignoring the cells, is that any three sites are linked into a triangle if the circumscribed circle has no site in its interior.)

As described in [3], we assign to each link (between sites $p_{i}$ and $p_{j}$ ) a conductance $\lambda_{i j}=b_{i j} / \ell_{i j}$, where $\ell_{i j}$ is the length of the link and $b_{i j}$ is the length of the boundary between the cells containing $p_{i}$ and $p_{j}$.

Let us call a link "exterior" if the corresponding cell boundary terminates on the circumference of the circle of radius $R$. The exterior links form a polygon enclosing all sites except those on its vertices. We call the latter "exterior sites" (see Fig. 1).

To define $g$, we set the potential $V=0$ on all exterior sites, $V=V_{0}$ at $p_{0}$, and identify $V_{0}^{2} / g$ with the total power dissipated in all the conducting links. (An exception arises when $p_{0}$ is exterior, very improbable when $\varrho R^{2} \gg 1$. In this case we define $g=0$. Since we seek an upper bound for $\langle g\rangle$, this case cannot harm the argument.) 


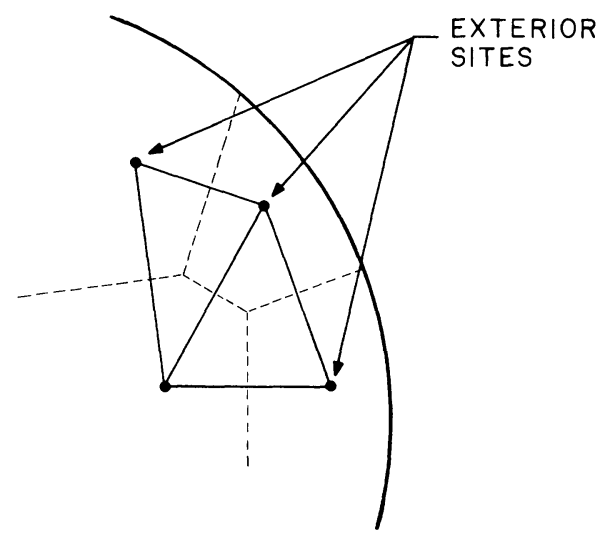

Fig. 1. A section of the lattice near the bounding circle, showing three exterior sites and two exterior links

We now define $\langle g\rangle$ as the average of $g$ over the ensemble of lattices described above.

\section{Partition of Problem}

In this section we state and prove a theorem which shows that $g$ cannot exceed a quantity determined by the immediate neighborhood of $p_{0}$.

Theorem 1. For $g$ defined as in the previous section, we have

$$
g \leqq g_{1}+g_{2},
$$

where

$$
\begin{aligned}
& g_{1}=\frac{2 \ell}{h}, \\
& g_{2}=\frac{1}{2 \pi} \ln \frac{R}{r} .
\end{aligned}
$$

Here $\ell$ is the length of a particular link to $p_{0}, h$ is the altitude of one of the triangles based on that link, and $r$ is the smallest altitude of a triangle containing $p_{0}$, measured from $p_{0}$ to the opposite side. To remove any ambiguity, we state that the triangle determining $h$ is the one on the right-hand side of $\ell$, looking from $p_{0}$, and that the link determining $\ell$ is the one giving the largest ratio $\ell / h$ (see Fig. 2).

Proof. We have already specified that

$$
\mathscr{P}=V_{0}^{2} / g
$$

where $\mathscr{P}$ is the power dissipated in all the links when the potential is set to $V_{0}$ at $p_{0}$ and to zero at all exterior sites. Under these conditions the potential at sites linked directly to $p_{0}$ will assume various values. Let $V_{1}$ be the lowest of these neighbor 


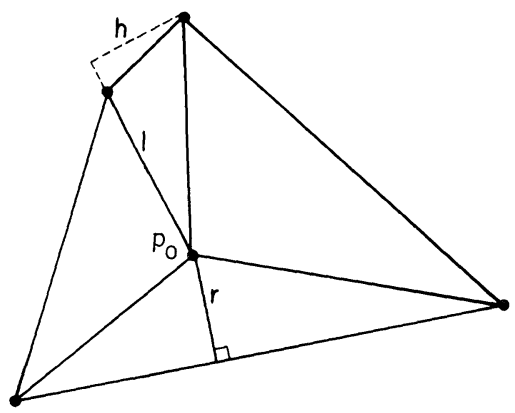

Fig. 2. A typical immediate polygon showing the two triangles from which $\ell, h, r$ of Eqs. (2) and (3) are determined

potentials. We shall prove that

$$
\mathscr{P} \geqq \frac{\left(V_{0}-V_{1}\right)^{2}}{g_{1}}+\frac{V_{1}^{2}}{g_{2}},
$$

where $g_{1}, g_{2}$ are given by (2) and (3). By minimizing the right side of (5) with respect to $V_{1}$, it follows that

$$
\mathscr{P} \geqq \frac{V_{0}^{2}}{g_{1}+g_{2}},
$$

which implies (1).

To prove (5), we subject the configuration to a series of modifications, each of which can only decrease or leave unchanged the total power dissipated.

Step 1. We appeal to Theorem 3 of [3]. This states that $\mathscr{P}$ remains unchanged if we replace the conducting links by a continuous medium of conductivity 1 filling up all the triangles ${ }^{1}$, provided that we constrain the potential as follows: at each site the potential must have the same value that it attained in the network problem, and along each link the potential must vary linearly. (Under these conditions the field within each triangle will assume a constant vector value, without further constraint.)

Note that this theorem applies only for two dimensions and only when the conductances are as prescribed in [3]. Strictly, we should have defined the conductance of our exterior links in a peculiar way, to make the theorem exactly true, see Eqs. (A1)-(A4) of [3]. But we have set the potential to zero on all exterior sites. Therefore no current flows through these links anyway, and their conductance has no effect. Hence no correction is necessary.

Step 2. We also fill up the space between the bounding circle and the exterior links with the same conducting medium. We set the potential to zero on the bounding circle. Then it is zero everywhere in this new space, so that no current flows here. Hence the total power is still $\mathscr{P}$.

1 Conductivity has the same units as conductance in two dimensions, and our conductances $\lambda_{i j}=b_{i j} / \ell_{i j}$ are dimensionless 


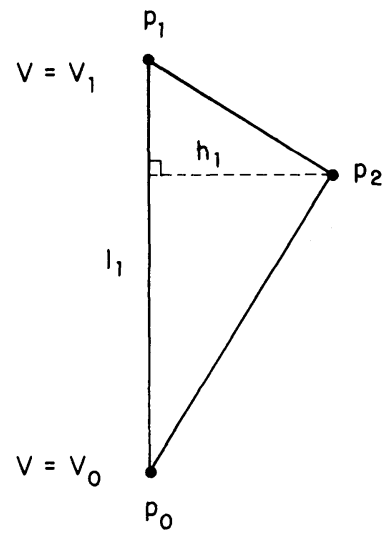

Fig. 3. The triangle selected to give a lower bound for $\mathscr{P}_{1}$

Step 3. The sites directly linked to $p_{0}$ are vertices of a polygon which we may call the "immediate polygon". We now relax all constraints on the potential except those on the interior and boundary of the immediate polygon, and on the circumference of the bounding circle. By a well-known theorem of current flow, the potential between the immediate polygon and the bounding circle will adjust itself so as to minimize the power. Hence the total power is not more than $\mathscr{P}$.

We are now in a position to write

$$
\mathscr{P} \geqq \mathscr{P}_{1}+\mathscr{P}_{2} .
$$

Here $\mathscr{P}_{1}$ is the power dissipated inside the immediate polygon, where the field is constant within each triangle, the potential at $p_{0}$ is $V_{0}$, the potential on the boundary varies linearly along each link but is otherwise unknown to us, and $\mathscr{P}_{2}$ is the power dissipated outside the immediate polygon, where the potential vanishes at the bounding circle, is continuous at the boundary of the immediate polygon with the potential used to determine $\mathscr{P}_{1}$, but is otherwise unconstrained. From now on we consider $\mathscr{P}_{1}$ and $\mathscr{P}_{2}$ separately.

Of the sites linked to $p_{0}$, let $p_{1}$ have the lowest potential $V_{1}$. Let $\ell_{1}$ be the distance from $p_{0}$ to $p_{1}$, and let $h_{1}$ be the perpendicular distance from the line $\overline{p_{0} p_{1}}$ to the site $p_{2}$, which is the vertex of the immediate polygon next after $p_{1}$ in a clockwise direction.

Let us now choose our coordinate system so that the $y$-axis is parallel to $\overline{p_{0} p_{1}}$. Then the $y$-component of the field in the triangle $p_{0} p_{1} p_{2}$ is known; it is

$$
E_{y}=\left(V_{0}-V_{1}\right) / \ell_{1}
$$

(see Fig. 3). The power dissipated in this triangle is $E^{2} A$, where $\mathbf{E}$ is the total field and $A=\frac{1}{2} \ell_{1} h_{1}$. Since $E^{2} \geqq E_{y}^{2}$, and this triangle is only a part of the immediate polygon, we have

$$
\begin{aligned}
\mathscr{P}_{1} \geqq E^{2} A & \geqq\left[\left(V_{0}-V_{1}\right) / \ell_{1}\right]^{2} \cdot \frac{1}{2} \ell_{1} h_{1} \\
& =\left(V_{0}-V_{1}\right)^{2} \cdot h_{1} / 2 \ell_{1} .
\end{aligned}
$$


Now, we are not guaranteed that the triangle $p_{0} p_{1} p_{2}$ is the one used to define $g_{1}$. But the choice of the latter triangle is such that

$$
\ell / h \geqq \ell_{1} / h_{1},
$$

and therefore (9) implies

$$
\mathscr{P}_{1} \geqq\left(V_{0}-V_{1}\right)^{2} h / 2 \ell=\frac{\left(V_{0}-V_{1}\right)^{2}}{g_{1}} .
$$

To analyze $\mathscr{P}_{2}$, we carry out some additional modifications.

Step 4 . We lower the potential to $V_{1}$ everywhere on the boundary of the immediate polygon.

It is intuitively evident that this reduces the power outside the polygon, but we can sketch a proof. Let $\mathbf{E}=\mathbf{E}_{a}+\mathbf{E}_{b}$, where $\mathbf{E}$ is the field determining $\mathscr{P}_{2}$ (present before this step), $\mathbf{E}_{a}$ is the field present after this step, and $\mathbf{E}_{b}$ is the difference. Then $\mathbf{E}_{b}$ is the field that would be present if the potential on the immediate polygon were replaced by its excess over $V_{1}$. Since this excess is never negative, the field lines of $\mathbf{E}_{b}$ can arise only on the polygon, although they may terminate either on the polygon or on the bounding circle.

We may now write

$$
\mathscr{P}_{2}=\mathscr{P}_{2}^{a a}+2 \mathscr{P}_{2}^{a b}+\mathscr{P}_{2}^{b b}
$$

in an obvious notation. Since $\mathscr{P}_{2}^{b b}$ is positive definite, we need only show that $\mathscr{P}_{2}^{a b} \geqq 0$. Explicitly

$$
\mathscr{P}_{2}^{a b}=\int \mathbf{E}_{a} \cdot \mathbf{E}_{b}=\int \mathbf{E}_{a} \cdot \mathbf{J}_{b},
$$

where the current density $\mathbf{J}$ is equal to the field. The area under consideration can be divided into tubes of flux of $\mathbf{J}_{b}$. Within each tube a constant current $d I_{b}$ flows from source to sink. The contribution of this tube to $\mathscr{P}_{2}^{a b}$ is

$$
d \mathscr{P}_{2}^{a b}=d I_{b} \int_{\text {source }}^{\text {sink }} \mathbf{E}_{a} \cdot d \ell=d I_{b} \cdot \Delta V_{a},
$$

where $\Delta V_{a}=0$ if the tube begins and ends on the polygon, or $\Delta V_{a}=V_{1} \geqq 0$ if it begins on the polygon and ends on the circle. Hence (14) is never negative, and so

$$
\mathscr{P}_{2} \geqq \mathscr{P}_{2}^{a a}
$$

as claimed.

Step 5. We fill in the space between the immediate polygon and a circle of radius $r$, centered at $p_{0}$, with the same conducting medium. (This is the largest such circle that remains inside the polygon.) Throughout this new space we set $V=V_{1}$, so that no current flows and no power is added. The power between polygon and circle is still $\mathscr{P}_{2}^{a a}$.

Step 6. We maintain $V=V_{1}$ on the circumference of the inner circle (radius $r$ ) and $V=0$ on the circumference of the bounding circle (radius $R$ ) but relax any constraint on the space between. By an elementary calculation the power is now 


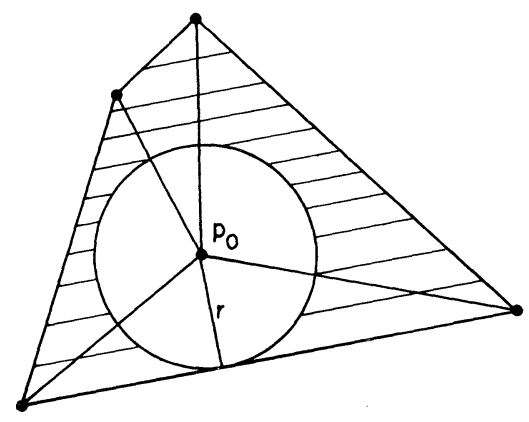

Fig. 4. The immediate polygon of Fig. 2. The shaded region is the new space filled up in Step 5. $V=V_{1}$ throughout this region

$V_{1}^{2} / g_{2}$, where $g_{2}$ is given by (3). But this is the minimum value for the power with potential fixed on the two circles. Therefore

$$
V_{1}^{2} / g_{2} \leqq \mathscr{P}_{2}^{a a} \text {. }
$$

Combining (7), (11), (15), and (16), we obtain (5). This completes the proof of Theorem 1.

\section{Upper Bound on $\left\langle g_{1}\right\rangle$}

We start by establishing two lemmas.

Lemma 1. Suppose that a variable $\beta$ is distributed between 0 and $\infty$ with probability $P(\beta) d \beta$, where $\int_{0}^{\infty} P(\beta) d \beta=1$. Suppose that $f(\beta)$ is a decreasing function of $\beta$ for $0<\beta<\propto$, and that another function $\tilde{P}$ is given so that

$$
P(\beta) \leqq \tilde{P}(\beta), \quad 0<\beta<\infty .
$$

Then

$$
\int_{0}^{\infty} f(\beta) P(\beta) d \beta \leqq \int_{0}^{\bar{\beta}} f(\beta) \tilde{P}(\beta) d \beta,
$$

where $\bar{\beta}$ is defined by

$$
\int_{0}^{\bar{\beta}} \tilde{P}(\beta) d \beta=1 .
$$

Proof. Let $Q$ and $\tilde{Q}$ be two functions such that

$$
\begin{aligned}
& \int_{0}^{Q(x)} P(\beta) d \beta=x, \\
& \int_{0}^{\tilde{Q}(x)} \tilde{P}(\beta) d \beta=x
\end{aligned}
$$




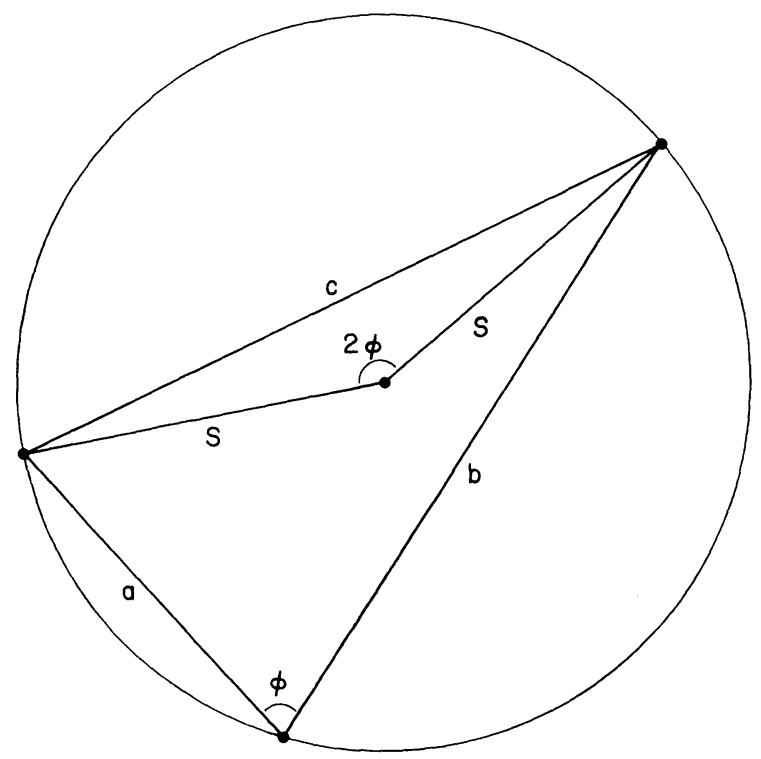

Fig. 5. The triangle and circumscribed circle entering into Lemma 2

for $0<x<1$. Then from (17) it follows that

$$
Q(x) \geqq \tilde{Q}(x)
$$

for $0<x<1$. Now (18) can be rewritten as

$$
\int_{0}^{1} f(Q(x)) d x \leqq \int_{0}^{1} f(\tilde{Q}(x)) d x,
$$

which follows from (22) since $f$ is a decreasing function.

Lemma 2. If the sides of a triangle are $a, b, c$ and the altitude to the base $c$ is $h$, then the circumscribed circle has radius

$$
s=\frac{a b}{2 h}
$$

Proof. Let $\phi$ be the angle between $a$ and $b$. The area of the triangle can be expressed in two ways:

$$
\frac{1}{2} \operatorname{ch}=\frac{1}{2} a b \sin \phi .
$$

But $c$ is a chord of the circumscribed circle, subtending an angle $2 \phi$ at the center, by an elementary theorem of geometry (see Fig. 5). Therefore

$$
c=2 s \sin \phi \text {. }
$$

Combining (25) and (26), we obtain (24). 
We now write (2) as

$$
g_{1}=\frac{4}{\beta}
$$

where

$$
\beta=\frac{2 h}{\ell}
$$

For any triangle $p_{0} p_{1} p_{2}$, where the vertices have been written in clockwise order starting with $p_{0}$, we infer a value of $\beta$ from (28) by taking $\ell$ as the length of $\overline{p_{0} p_{1}}$ and $h$ as the altitude from $p_{2}$. Then

$$
\left\langle g_{1}\right\rangle=\int_{0}^{\infty} \frac{4}{\beta} P(\beta) d \beta,
$$

where $P(\beta) d \beta$ is the probability that the largest value of $2 h / \ell$ obtained from a triangle of the lattice including $p_{0}$ lies between $\beta$ and $\beta+d \beta$.

We shall calculate a function $\tilde{P}(\beta)$, where $\tilde{P}(\beta) d \beta$ is the probability that some triangle of the lattice including $p_{0}$ gives $2 h / \ell$ between $\beta$ and $\beta+d \beta$. Obviously $\tilde{P}(\beta) \geqq P(\beta)$, and (27) gives $g_{1}$ as a decreasing function of $\beta$. Therefore Lemma 1 applies, and we shall have

$$
\left\langle g_{1}\right\rangle \leqq \int_{0}^{\bar{\beta}} \frac{4}{\beta} \tilde{P}(\beta) d \beta
$$

with $\bar{\beta}$ given by (19).

To find $\tilde{P}(\beta)$, we note that the probability of finding $p_{1}$ so that $\overline{p_{0} p_{1}}$ lies between $\ell$ and $\ell+d \ell$ is $\varrho \cdot 2 \pi \ell d \ell$. Then taking cartesian coordinates $(h, y)$ for $p_{2}$, with $h$ measured perpendicular to $\overline{p_{0} p_{1}}$ and $y$ along $\overline{p_{0} p_{1}}$, and $h=y=0$ at the midpoint of $\overline{p_{0} p_{1}}$, the probability of finding $p_{2}$ in an area $d h d y$ is $\varrho d h d y$. Finally the probability that $p_{0} p_{1} p_{2}$ is a triangle of the lattice is just the probability $e^{-\varrho \pi s^{2}}$ that the circumscribed circle has empty interior. (This is the probability of no events in a Poisson distribution in which the average number of events is $\varrho \pi s^{2}$.) Here $s$ is the radius of the circle and by Lemma 2 is given by

$$
s=\sqrt{h^{2}+\left(y-\frac{1}{2} \ell\right)^{2}} \cdot \sqrt{h^{2}+\left(y+\frac{1}{2} \ell\right)^{2}} / 2 h .
$$

The product of these probabilities is

$$
\varrho^{2} \cdot 2 \pi \ell d \ell d h d y e^{-\varrho \pi s^{2}} .
$$

Now let us define

$$
\alpha=\frac{2 y}{\ell}, \quad \beta=\frac{2 h}{\ell} .
$$

We then have

$$
s^{2}=\ell^{2} K(\alpha, \beta)
$$


where

$$
K=\left[\beta^{2}+(\alpha+1)^{2}\right]\left[\beta^{2}+(\alpha+1)^{2}\right] / 16 \beta^{2} .
$$

Then integrating (32) over $\ell$ and $y$, with fixed $\beta$, we have

$$
\begin{aligned}
\tilde{P}(\beta) d \beta & =\varrho^{2} \cdot \int_{0}^{\infty} 2 \pi \ell d \ell \cdot \frac{1}{2} \ell d \beta \int_{-\infty}^{\infty} \frac{1}{2} \ell d \alpha e^{-\varrho \pi \ell^{2} K} \\
& =d \beta \cdot \varrho^{2} \frac{\pi}{2} \int_{0}^{\infty} \ell^{3} d \ell \int_{-\infty}^{\infty} e^{-\varrho \pi \ell^{2} K} d \alpha \\
& =d \beta \cdot \frac{1}{4 \pi} \int_{-\infty}^{\infty} \frac{d \alpha}{K^{2}}
\end{aligned}
$$

with $K$ given by (35).

A contour integration gives

$$
\int_{-\infty}^{\infty} \frac{d \alpha}{\left[\beta^{2}+(\alpha-1)^{2}\right]^{2}\left[\beta^{2}+(\alpha+1)^{2}\right]^{2}}=\frac{\pi}{16 \beta^{3}} \cdot \frac{1+5 \beta^{2}}{\left(1+\beta^{2}\right)^{3}},
$$

and combining this with (36) and (35), we have

$$
\tilde{P}(\beta)=4 \beta\left(1+5 \beta^{2}\right) /\left(1+\beta^{2}\right)^{3}
$$

Substituting this into (19) we obtain

$$
\bar{\beta}=5^{-1 / 4} \text {. }
$$

From (30) we then have

$$
\begin{aligned}
\left\langle g_{1}\right\rangle & \leqq 16\left[\tan ^{-1} \bar{\beta}+\frac{\bar{\beta}^{3}}{\left(1+\bar{\beta}^{2}\right)^{2}}\right]=16\left[\tan ^{-1} 5^{-1 / 4}+\frac{5^{1 / 4}}{(\sqrt{5}+1)^{2}}\right] \\
& \cong 11.7
\end{aligned}
$$

\section{Upper Bound on $\left\langle g_{2}\right\rangle$}

The distance $r$ appearing in (3) is the altitude of a lattice triangle $p_{0} p_{1} p_{2}$, dropped from $p_{0}$ as vertex. It is specified that $r$ is the least such altitude, but we shall drop that restriction, thereby over-estimating the probability. Thus we shall study $P_{1}(r)$, where $P_{1}(r) d r$ is the probability of finding $a$ triangle whose altitude from $p_{0}$ is between $r$ and $r+d r$, and

$$
P_{1}(r) \geqq P(r)
$$

where $P(r) d r$ is the true probability distribution of $r$.

Let us assign cartesian coordinates $\left( \pm r, y_{1}\right)$ to $p_{1}$ and $\left( \pm r, y_{2}\right)$ to $p_{2}$. We remove ambiguity by requiring $\left|y_{1}\right| \leqq y_{2}$ (see Fig. 6). To find the probability associated with a given triplet $\left(r, y_{1}, y_{2}\right)$ we first locate $p_{2}$ with respect to $p_{1}$; this involves infinitesimal probability $2 \pi\left|y_{2}-y_{1}\right| d\left(y_{2}-y_{1}\right)$ since the line $\overline{p_{1} p_{2}}$ can be any direction. We then fix our coordinate system so that $\overline{p_{1} p_{2}}$ is parallel to the $y$-axis, with $p_{2}$ above $p_{1}$. Translating $\overline{p_{1} p_{2}}$ so as to locate $p_{1}$ with respect to $p_{0}$, we 

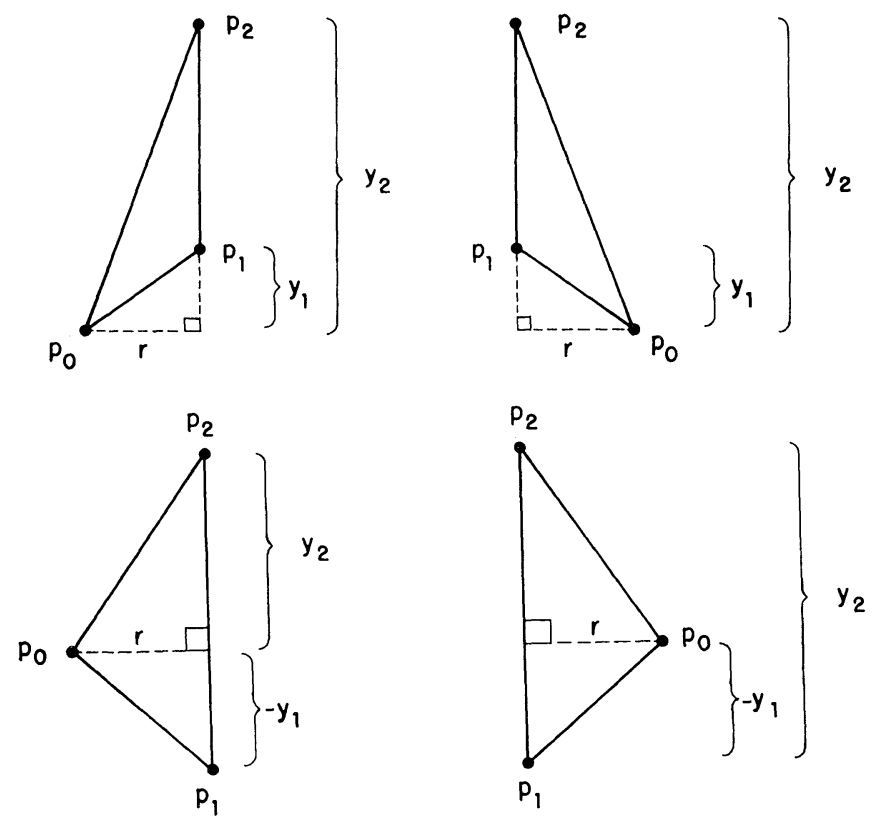

Fig. 6. The triangle determining $g_{2}$, with the coordinates $r, y_{1}, y_{2}$. Four allowed cases are shown. Note that $y_{2}>\left|y_{1}\right|$ in all cases

find a second infinitesimal probability $2 \varrho d r d y_{1}$, where the factor 2 accounts for the fact that $p_{0}$ may be on either side of $\overline{p_{1} p_{2}}$. Finally the circumscribed circle must be empty; the probability for this is $e^{-\varrho \pi s^{2}}$, where by Lemma 2 we have

$$
s=\sqrt{y_{1}^{2}+r^{2}} \sqrt{y_{2}^{2}+r^{2}} / 2 r .
$$

Assembling these factors, we have

$$
\begin{aligned}
P_{1}(r) & =\varrho^{2} \int_{-\infty}^{\infty} 2 d y_{1} \int_{\left|y_{1}\right|}^{\infty} d y_{2} 2 \pi\left(y_{2}-y_{1}\right) e^{-\varrho \pi s^{2}} \\
& =\varrho^{2} \int_{0}^{\infty} 2 d y_{1} \int_{y_{1}}^{\infty} d y_{2} 2 \pi \cdot 2 y_{2} e^{-\varrho \pi s^{2}} \\
& =8 \pi \varrho^{2} \int_{0}^{\infty} d y_{1} \int_{y_{1}}^{\infty} y_{2} d y_{2} e^{-\varrho \pi\left(y_{1}^{2}+r^{2}\right)\left(y_{2}^{2}+r^{2}\right) / 4 r^{2}} \\
& =4 \varrho \int_{0}^{\infty} d y_{1} \cdot \frac{4 r^{2}}{y_{1}^{2}+r^{2}} e^{-\varrho \pi\left(y_{1}^{2}+r^{2}\right)^{2} / 4 r^{2}} \\
& \leqq 4 \varrho \int_{0}^{\infty} d y_{1} \cdot \frac{4 r^{2}}{y_{1}^{2}+r^{2}}=8 \pi \varrho r .
\end{aligned}
$$

Combining (43) with (41), we have

$$
P(r) \leqq \tilde{P}(r)=8 \pi \varrho r .
$$


Since (3) is a decreasing function of $r$, Lemma 1 applies with $r$ in place of $\beta$. From (19) we have

$$
\bar{r}=\sqrt{1 / 4 \pi \varrho}
$$

and (18) becomes

$$
\begin{aligned}
\left\langle g_{2}\right\rangle & \equiv \int_{0}^{\infty} P(r) \frac{1}{2 \pi} \ln \frac{R}{r} d r \\
& \leqq \int_{0}^{\bar{r}} 8 \pi \varrho r \cdot \frac{1}{2 \pi} \ln \frac{R}{r} d r \\
& =\frac{1}{2 \pi} \ln \frac{R}{\bar{r}}+\varrho \bar{r}^{2} \\
& =\frac{1}{4 \pi}\left[\ln \left(4 \pi \varrho R^{2}\right)+1\right] .
\end{aligned}
$$

\section{Results and Discussion}

We have proved rigorously that the ensemble average of the resistance between a site at the origin and a bounding circle of radius $R$, as defined more fully in Sect. 2 and references therein, is bounded above by

$$
\begin{aligned}
\langle g\rangle & \leqq \frac{1}{4 \pi}\left[\ln \left(4 \pi \varrho R^{2}\right)+1\right]+16\left[\tan ^{-1} 5^{-1 / 4}+\frac{5^{1 / 4}}{(\sqrt{5}+1)^{2}}\right] \\
& \cong \frac{1}{4 \pi} \ln \left(\varrho R^{2}\right)+12.0 .
\end{aligned}
$$

No doubt the second term is much too large. In estimating $\mathscr{P}_{1}$ in Sect. 3 , we discarded all but one of the triangles of the immediate polygon; in typical cases this means we underestimated $\mathscr{P}_{1}$ by 5 or 6 times and consequently (2) gives 5 or 6 times too large a value of $g_{1}$. Of course, in exceptional lattices this reasoning may break down; but anyone who tries to construct a lattice in which $\mathscr{P}_{1}$ is as small as permitted by (11) and (40) will run into difficulties, unless he is cleverer than we. The last escape clause, however, precludes our offering a rigorous bound much smaller than (47).

We may compare (47) with numerical averages computed by Mr. H. C. Ren, who obtains [4]

$$
\langle g\rangle \cong 0.90
$$

on a square $(L \times L)$ lattice with boundary conditions periodic in one direction, antiperiodic in the other, and $\varrho L^{2}=10^{4}$.

The continuum theory with such boundary conditions gives the potential at small distances $r$, due to a unit current source, as

$$
V(r) \cong \frac{1}{2 \pi} \ln \frac{\sqrt{2} L}{K r},
$$


where

$$
K=\int_{0}^{1} \frac{d x}{\sqrt{1-x^{2}} \sqrt{1-x^{2}}} \cong 1.8541 .
$$

In the circular configuration considered in the present paper, the continuum theory gives

$$
V(r)=\frac{1}{2 \pi} \ln \frac{R}{r}
$$

Therefore we can compare the two theories by replacing $R \rightarrow \sqrt{2} L / K$ or, in this case, $\sqrt{\varrho} R \rightarrow 10^{2} \cdot \sqrt{2} / 1.8541=76.27$. Thus $(48)$ can be written as

$$
\langle g\rangle \cong \frac{1}{2 \pi} \ln 76.27+0.21,
$$

where the second term corresponds to the second term 12.0 of (47).

\section{References}

1. Christ, N.H., Friedberg, R., Lee, T.D.: Random lattice field theory. Nucl. Phys. B202, 89-125 (1982)

2. Christ, N.H., Friedberg, R., Lee, T.D.: Gauge theory on a Random lattice. Nucl. Phys. B210, 310-336 (1982)

3. Christ, N.H., Friedberg, R., Lee, T.D. : Weights of links and plaquettes in a Random lattice. Nucl. Phys. B210, 337-346 (1982)

4. Christ, N.H., Friedberg, R., Lee, T.D., Ren, H.C.: Numerical computations on a Random lattice. Columbia University preprint CU-TP-245

Communicated by A. Jaffe

Received September 13, 1982; in revised form December 21, 1982 
